Mater. Res. Soc. Symp. Proc. Vol. 1774 (C) 2015 Materials Research Society

DOI: 10.1557/opl.2015.745

\title{
Lithium Dendrite Inhibition on Post-Charge Anode Surface: The Kinetics Role
}

\author{
Asghar Aryanfar ${ }^{1 *}$,Tao Cheng ${ }^{2}$, Boris V. Merinov ${ }^{2}$, William A. Goddard $\mathrm{III}^{2}$, Agustin J Colussi ${ }^{1}$ \\ and Michael R. Hoffmann ${ }^{1}$ \\ ${ }^{1}$ Linde Center for Global Environmental Science, ${ }^{2}$ Materials and Process Simulation Center \\ California Institute of Technology, 1200 E California Blvd, Pasadena, CA, 91125.
}

\begin{abstract}
We report experiments and molecular dynamics calculations on the kinetics of electrodeposited lithium dendrites relaxation as a function of temperature and time. We found that the experimental average length of dendrite population decays via stretched exponential functions of time toward limiting values that depend inversely on temperature. The experimental activation energy derived from initial rates as $\mathrm{E}_{\mathrm{a}} \sim 6-7 \mathrm{kcal} / \mathrm{mole}$, which is closely matched by MD calculations, based on the ReaxFF force field for metallic lithium. Simulations reveal that relaxation proceeds in several steps via increasingly larger activation barriers. Incomplete relaxation at lower temperatures is therefore interpreted a manifestation of cooperative atomic motions into discrete topologies that frustrate monotonic progress by 'caging'.
\end{abstract}

Keywords: Lithium Dendrites, Kinetics, Activation Energy, Annealing.

\section{INTRODUCTION}

Wireless revolution and need for harnessing intermittent renewable energy sources, has created an exponential demand for energy storage devices such as batteries that require longlasting storage capacity and high-power delivery during last decade [1]. Lithium $\left(\mathrm{Li}^{0}\right)$, particularly, anode candidate material with an ideal energy density of $3862 \mathrm{mAh} / \mathrm{g}$, could drastically satisfy this demand. However, due to it relatively low surface energy, it has very high propensity to grow dendrites during consecutive recharging. This phenomenon eventually leads to short-circuiting, overheating the cell and possible ignition of the organic electrolyte as well as creating isolated 'dead lithium' crystals. [2]

The current reports have investigated the effect of charging method, [3, 4] current density [5-7], electrode surface morphology [8-10], solvent and electrolyte chemical composition [1113],electrolyte concentration [5, 14] on dendrite growth. Other methods include the use of powder electrodes [15] and adhesive polymers[16]. Recent studies have tried to explain the dendrite evolution mechanism [17] and have offered impurities as dendrite initiation drive [18, 19]. Current modeling frameworks involve simplifying assumptions that may have fallen short of capturing the comprehensive essentials of dendrite growth. [7,20,21]

* Corresponding Author: aryanfar@caltech.edu, Tel: +1 (626) 395-8736, Fax: +1 (626) 395-8535 
Although the ongoing research tends to extend the battery energy density by developing Lithium-Air and lithium-Sulfur batteries, the dendrite problem remains as a challenging issue in all kinds of rechargeable batteries. [22, 23]

Temperature is a highly accessible parameter with foremost important effect in kinetics. It has been found that cycling at higher temperatures (from -500C up to $40 \mathrm{oC}$ ) can, on average, cause more frequent short-circuiting events up to a factor of 2 [24]. Other results show that the increasing cell temperature enhances the ionic mobilities in favor of dendritic inception and growth [25]. [5, 26] reported that the higher temperatures extends ion depletion layer length which is in agreement with temperature dependence of reaction rates[6]. [27] also pointed out that the probability of ionic reduction in the electrode surface correlates directly to the temperature. In contrast,[28] found that imposing higher temperatures reduces dendrite growth rate relatively to the electrode surface, and could results in more uniform deposition. Although all those approaches are helpful, it is apparent that further progress in tackling this crucial issue should accrue from a full understanding of the dynamics of dendrite growth on Li-metal electrodes. [12, 29, 30]

In our recent work, we have found that applying higher anodic temperatures has a destructive effect for growing dendrites during charging periods [31] and show experimental evidence that higher temperatures during electrodeposition can reduce lithium dendrites to a considerable amount. Acquiring the inherent strctutral properties of lithium dendrties is one of hardest problems in the literature and obtaining the energy barrier for the annealing process would represent the dominant coodination number in the dendrite structure. In this paper, we characterize the post-charge anneliang of the electrodeposited lithium dendrites and we explain reasons leading to such reduction in details from QM insights.

\section{EXPERIMENTAL}

We have used unique manually-made cells for in situ observation and measurement of electrolytic $\mathrm{Li}^{0}$ deposits (Fig. 1-a). The cell components and details of the fabrication process have been described before.[32] Round disk electrodes $\left(\right.$ Area $\left.=1.6 \mathrm{~cm}^{2}\right)$ were punched from a $\mathrm{Li}^{0}$ foil (Aldrich, $99.9 \%, 0.38 \mathrm{~mm}$ thick) that had been cleaned of oxide layers by scraping with a blade and dimethyl carbonate (DMC) in a glove box sparged with argon $\left(\mathrm{H}_{2} \mathrm{O}, \mathrm{O}_{2}<0.5 \mathrm{ppm}\right)$. The disk electrodes were mounted on silicone gaskets and pressed against a polymethylmethacrylate (PMMA) electrode separator that kept them $\mathrm{L}=3.175 \mathrm{~mm}$ apart. The electrolyte, 1 $\mathrm{M} \mathrm{LiClO}_{4}$ (Aldrich, battery grade, $99.99 \%$, dried for 24 hours at $90{ }^{\circ} \mathrm{C}$ under vacuum) solution in propylene carbonate (PC) (Aldrich, 99.7\% Anhydrous) was injected into the cell, whereupon the opening was sealed with a Teflon plug. 


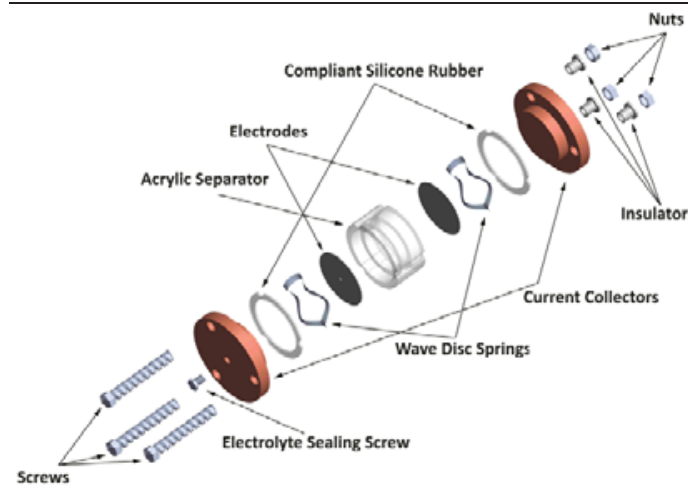

a

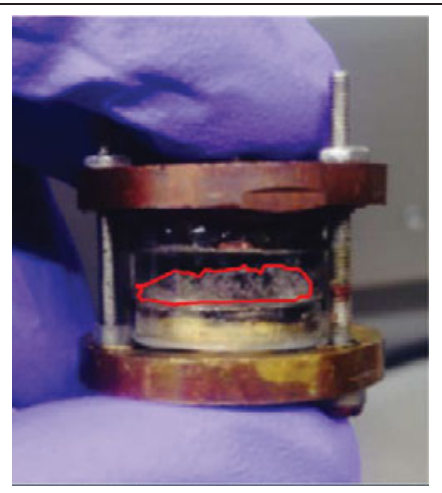

b

Fig. 1: (a) Component-level view of the cell. (b) Dendrites observed by naked eye (red enclosure) from the PMMA

separator.

We prepared four oil baths of different thermostated temperatures, using the beakers in the top of hot plates. Simultaneously, four cells were fabricated with the aforementioned procedure and were charged at the rate of $\mathrm{i}=2 \mathrm{~mA} / \mathrm{cm}^{2}$ for 24 hours $(\mathrm{Q}=48 \mathrm{mAh})$ in ambient condition using Biologic instruments (SP-50, VSP). The cells were disconnected, rinsed with isopropyl alcohol and the initial optical observations were done on the post-charge anode surface.

Subsequently, the cells were inserted in the oil baths temperatures and every 24 hours they were taken out and the optical observations on the dendrites are repeated in the cell perimeter. The dendrites lengths typically spanned from $200 \mu \mathrm{m}$ to $3000 \mu \mathrm{m}$ enabling to observe them with naked eye (Fig. 1-b). The lengths and multiplicities $\left[\lambda_{i}, p_{i}\right]$ of the 45 dendrites were measured in series of experiments. The figure of merit is defined as the average weighted lengths of measured dendrites normalized to interelectrode distance $L$ :

$$
\bar{\lambda}(T, t)=\frac{1}{L} \sum_{i=1}^{n} \lambda_{i} p_{i}
$$

where

$$
\sum_{i=1}^{n} p_{i}=1
$$




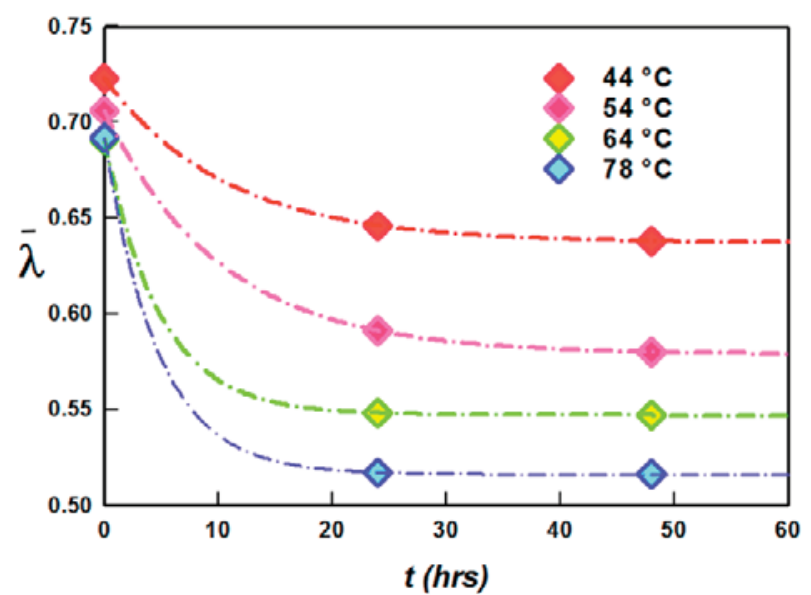

Fig. 2: Average dendrite length $\bar{\lambda}$ versus bath temperature for 4 various bath temperatures during 48 hours postcharge period.

\section{SIMULATIONS}

We have used the Reactive Force Field (ReaxFF) for simulating and verification of experimental results which is based on bond order [33] and we have trained reactive force field model for lithium subsequently ${ }^{1}$. [34]

Due to amorphous nature of lithium dendrites we have considered an ideal case of initial pyramid morphology that consists of 15 layers of Li bulk $(7 \times 7 \times 15)$ and 6 layers of Li pyramid leading to single atom tip (Fig. 3). The size in $\mathrm{x}$ and $\mathrm{y}$ direction is $24.57 \AA$ and in $\mathrm{z}$ direction is $38.61 \AA$. A $30 \AA$ vacuum space is built to avoid the interactions between the slab and its imagines. Since nano-scale structures usually exhibit very low melting point comparing with that in bulk phase, we fixed the bottom ten layers to avoid the unrealistic melting.

\footnotetext{
${ }^{1}$ Simulation parameters available upon request.
} 


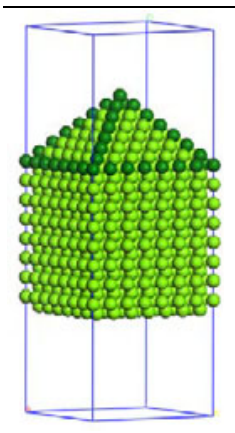

Fig. 3: The simulation pyramid tip model. (24.57 $\AA 24.57 \AA$ x $38.61 \AA$ )

Using the optimized structure we relax the $\mathrm{x}$ and $\mathrm{y}$ direction at $50 \mathrm{~K}$ to adjust the pressure to 1 bar by expending or compressing the box in $\mathrm{x}$ and $\mathrm{y}$ directions. The obtained structures were then heated up to target temperatures, $350 \mathrm{~K}, 400 \mathrm{~K}$ and $450 \mathrm{~K}$ during 200 ps NVT (i.e. constant number of atoms, volume and temperature) calculations, 0.25 fs time step, were carried out at evaluated temperature using Nose hover temperature control with a damping constant of $25 \mathrm{fs}$. [35] The simulation parameters are given in Table 1.

\begin{tabular}{|l|l|}
\hline $\mathrm{x}, \mathrm{y}$ dimension $(\AA)$ & 24.57 \\
\hline $\mathrm{z}$ dimension $(\AA)$ & 38.61 \\
\hline Cube layers & $7 \mathrm{x} 7 \mathrm{x} 15$ \\
\hline Tip layers & 15 \\
\hline Domain width $(\AA)$ & $24.57 \times 24.57$ \\
\hline Domain height $(\AA)$ & $10.53 \AA$ \\
\hline Target temperatures $(\mathrm{K})$ & $350,400,450(\sim$ melting point $)$ \\
\hline Simulation time $(\mathrm{ps})$ & 200 \\
\hline Simulation time step $(\mathrm{fs})$ & 0.25 \\
\hline Damping constant $(\mathrm{fs})$ & 12.5 \\
\hline
\end{tabular}

Table 1: Model parameters

\section{DISCUSSION}

We interpret that the degradation (reduction) of lithium dendrites follows the first-order kinetics and we ascribe the following:

$\bar{\lambda}(T, t)=\lambda_{\infty}+a \exp (-k(T) t)$

The reduction rate $k$ in our experiments shows to be affected by temperature $T$. Considering Arrhenius relation for kinetics, we have the following equation: 
$k(T)=k\left(T_{0}\right) \exp \left(\frac{E_{a}}{k_{B}}\left(\frac{1}{T}-\frac{1}{T_{0}}\right)\right)$

Hence by plotting the $\log (k)$ versus temperature reciprocal $1 / T$ we can interpret the exponential coefficient. (Fig. 4)

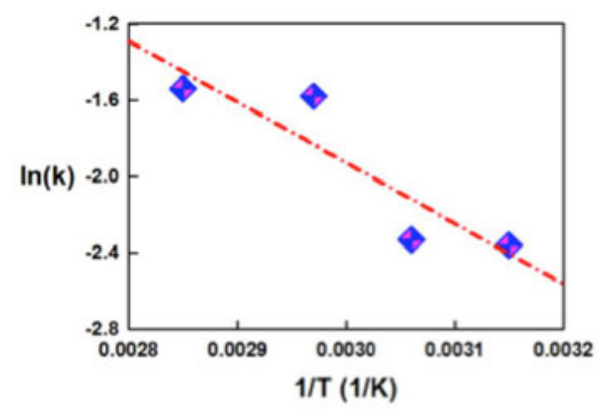

(a)

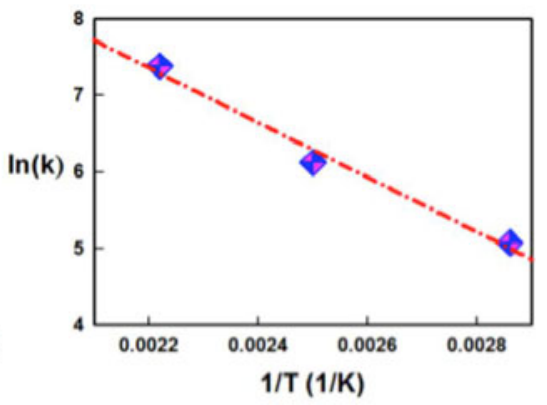

(b)

Fig. 4: Interpolation of experimental (a) and computational (b) annealing of dendrites versus reciprocal of temperature with first order Arrhenius kinetics. The effective activation energies $E_{a}$ are $6.3 \pm 2.1$ kcal $/ \mathbf{m o l e}$ and $7.1 \pm 0.9 \mathrm{kcal} / \mathrm{mole}$ respectively.

The experimental results revealed that typically shorter dendrites were formed at higher imposed temperatures (Fig. 2) which are consistent with previous studies [24, 28].It's also apparent that average dendrite length, $\bar{\lambda}$, versus time $t$ in certain temperature, $T$, converges to a constant. This trend is expected as letting the system sit for considerably higher time will saturate the system and all possible re-arrangements have been done.

Additionally, the calculated energy barrier is for surface diffusion $(9.715 \mathrm{kcal} / \mathrm{mole})$ in is lower than the bulk diffusion $(11.185 \mathrm{kcal} / \mathrm{mole})$. However, the difference is so small, it is highly possible that both of these mechanisms occur in the realist conditions.

\section{CONCLUSIONS}

In this paper, we observed the reduction of lithium dendrites in higher temperatures. We calculated the activation energy of 6-7 kcal/mole for dendrites annealing to more stable and toward crystalline phase. We verified the energy barrier via developing a ReaxFF model. The result is useful for understanding the structural propertie and kinetics Further work is under progress to understand the mechanism of reduction and evolved instabilities before dendrite collapse. 


\section{ACKNOWLEDGMENTS}

Authors gratefully acknowledge support from Bill and Melinda Gates Foundation Grant No. OPP1069500 on environmental sustainability and, in part, by Bosch Energy Research Network Grant No. 13.01.CC11.

\section{REFERENCES}

1. Armand, M. and J.M. Tarascon, Building better batteries. Nature, 2008. 451(7179): p. 652-657.

2. Aryanfar, A., et al., Quantifying the dependence of dead lithium losses on the cycling period in lithium metal batteries. Physical Chemistry Chemical Physics, 2014. 16(45): p. 24965-24970.

3. Aryanfar, A., et al., Dynamics of Lithium Dendrite Growth and Inhibition: Pulse Charging Experiments and Monte Carlo Calculations. The Journal of Physical Chemistry Letters, 2014. 5: p. 1721-1726.

4. Mayers, M.Z., J.W. Kaminski, and T.F. Miller III, Suppression of Dendrite Formation via Pulse Charging in Rechargeable Lithium Metal Batteries. The Journal of Physical Chemistry C, 2012. 116(50): p. 26214-26221.

5. Brissot, C., et al., In situ concentration cartography in the neighborhood of dendrites growing in lithium/polymer-electrolyte/lithium cells. Journal of the Electrochemical Society, 1999. 146(12): p. 4393-4400.

6. F. Orsini, A.D.P., B. Beaudoin, J.M. Tarascon, M. Trentin, N. Langenhuisen, E.D. Beer, P. Notten, In Situ Scanning Electron Microscopy (SEM) observation of interfaces with plastic lithium batteries. Journal of power sources, 1998. 76: p. 19-29.

7. Monroe, C. and J. Newman, Dendrite growth in lithium/polymer systems - A propagation model for liquid electrolytes under galvanostatic conditions. Journal of the Electrochemical Society, 2003. 150(10): p. A1377-A1384.

8. Liu, X.H., et al., Lithium fiber growth on the anode in a nanowire lithium ion battery during charging. Applied Physics Letters, 2011. 98(18).

9. Monroe, C. and J. Newman, The effect of interfacial deformation on electrodeposition kinetics. Journal of the Electrochemical Society, 2004. 151(6): p. A880-A886.

10. Nishida, T., et al., Optical observation of Li dendrite growth in ionic liquid. Electrochimica Acta, 2013.

11. Howlett, P.C., D.R. MacFarlane, and A.F. Hollenkamp, A sealed optical cell for the study of lithium-electrode electrolyte interfaces. Journal of Power Sources, 2003. 114(2): p. 277-284.

12. Schweikert, N., et al., Suppressed lithium dendrite growth in lithium batteries using ionic liquid electrolytes: Investigation by electrochemical impedance spectroscopy, scanning electron microscopy, and in situ Li-7 nuclear magnetic resonance spectroscopy. Journal of Power Sources, 2013. 228: p. 237-243.

13. Crowther, O. and A.C. West, Effect of electrolyte composition on lithium dendrite growth. Journal of the Electrochemical Society, 2008. 155(11): p. A806-A811. 
14. Brissot, C., et al., Dendritic growth mechanisms in lithium/polymer cells. Journal of Power Sources, 1999. 81: p. 925-929.

15. Seong, I.W., et al., The effects of current density and amount of discharge on dendrite formation in the lithium powder anode electrode. Journal of Power Sources, 2008. 178(2): p. 769-773.

16. Stone, G., et al., Resolution of the Modulus versus Adhesion Dilemma in Solid Polymer Electrolytes for Rechargeable Lithium Metal Batteries. Journal of The Electrochemical Society, 2012. 159(3): p. A222-A227.

17. Steiger, J., D. Kramer, and R. Mönig, Microscopic observations of the formation, growth and shrinkage of lithium moss during electrodeposition and dissolution. Electrochimica Acta, 2014.

18. Harry, K.J., et al., Detection of subsurface structures underneath dendrites formed on cycled lithium metal electrodes. Nat Mater, 2014. 13(1): p. 69-73.

19. Steiger, J., D. Kramer, and R. Monig, Mechanisms of dendritic growth investigated by in situ light microscopy during electrodeposition and dissolution of lithium. Journal of Power Sources, 2014. 261: p. 112-119.

20. Chazalviel, J.N., Electrochemical Aspects of the Generation of Ramified Metallic Electrodeposits. Physical Review A, 1990. 42(12): p. 7355-7367.

21. Zhang, H.-W., et al. Understanding and Predicting Li Dendrite Formation in Li-Ion Batteries: Phase Field Model. in Meeting Abstracts. 2014. The Electrochemical Society.

22. Goodenough, J.B. and Y. Kim, Challenges for rechargeable batteries. Journal of Power Sources, 2011. 196(16): p. 6688-6694.

23. Goodenough, J.B. and K.-S. Park, The Li-ion rechargeable battery: a perspective. Journal of the American Chemical Society, 2013. 135(4): p. 1167-1176.

24. Park, H.E., C.H. Hong, and W.Y. Yoon, The effect of internal resistance on dendritic growth on lithium metal electrodes in the lithium secondary batteries. Journal of Power Sources, 2008. 178(2): p. 765-768.

25. Diggle, J., A. Despic, and J.M. Bockris, The mechanism of the dendritic electrocrystallization of zinc. Journal of The Electrochemical Society, 1969. 116(11): p. 1503-1514.

26. Brissot, C., et al., Concentration measurements in lithium/polymer-electrolyte/lithium cells during cycling. Journal of Power Sources, 2001. 94(2): p. 212-218.

27. Bard, A.J. and L.R. Faulkner, Electrochemical methods: fundamentals and applications. 1980. 2 New York: Wiley, 1980.

28. Akolkar, R., Modeling dendrite growth during lithium electrodeposition at sub-ambient temperature. Journal of Power Sources, 2014. 246: p. 84-89.

29. Bhattacharyya, R., et al., In situ NMR observation of the formation of metallic lithium microstructures in lithium batteries. Nature Materials, 2010. 9(6): p. 504-510.

30. Harry, K.J., et al., Detection of subsurface structures underneath dendrites formed on cycled lithium metal electrodes. Nature materials, 2014. 13(1): p. 69-73.

31. Aryanfar, A., et al., Thermal relaxation of lithium dendrites. Physical Chemistry Chemical Physics, 2015. 17(12): p. 8000-8005.

32. Aryanfar, A., Method and device for dendrite research and discovery in batteries. 2014, US Patent App. 14/201,979. 
33. Chenoweth, K., A.C. van Duin, and W.A. Goddard, ReaxFF reactive force field for molecular dynamics simulations of hydrocarbon oxidation. The Journal of Physical Chemistry A, 2008. 112(5): p. 1040-1053.

34. Van Duin, A.C.T., et al., ReaxFF: a reactive force field for hydrocarbons. The Journal of Physical Chemistry A, 2001. 105(41): p. 9396-9409.

35. Martyna, G.J., D.J. Tobias, and M.L. Klein, Constant pressure molecular dynamics algorithms. The Journal of Chemical Physics, 1994. 101(5): p. 4177-4189. 